\title{
Measuring the angular emission of optical vortex beams from integrated devices
}

\author{
Lifeng Chen ${ }^{1}$, Jiangbo Zhu ${ }^{1,2}$, Michael J. Strain ${ }^{3,4}$, Laura Meriggi ${ }^{4}$, Martin L. Garcia ${ }^{1}$, Kenan Cicek ${ }^{1}$, \\ Huanlu Li ${ }^{1}$, Xinlun $\mathrm{Cai}^{2}$, Marc Sorel ${ }^{4}$, Siyuan $\mathrm{Yu}^{1,2}$ \\ 1. Photonics Group, Merchant Venturers School of Engineering, University of Bristol, UK \\ 2. State Key Laboratory of Optoelectronic Mat. and Technologies and School of Physics and Engineering, Sun Yatsen University, Guangzhou, China. \\ 3. Institute of Photonics, University of Strathclyde, Glasgow, G4 0NW, U.K \\ 4. School of Engineering, University of Glasgow, Glasgow, G12 8LT, U. K.
}

Orbital angular momentum (OAM) represents an additional degree of freedom of a light beam, to be added to the standard ones (e.g. polarization, wavelength) [1-2]. Previously, we have demonstrated an integrated device for OAM generation, in which the Whispering Gallery mode (WGM) of the micro-ring resonator to a propagating OAM mode by using an angular grating embedded within the resonator structure [3]. This integrated approach allows compact device footprint and OAM mode order selectivity with injection wavelength, which is highly desirable for telecommunications systems. To bring OAM closer to actual system implementations, several groups around the world are working on the development of optical fibres for propagation of multiplexed OAM modes. In order to achieve efficient coupling between OAM beams and OAM fibres, it is important to obtain the information of the divergence angle of the OAM beams which should be compatible with the N.A. of the fibres. In this work, we use Fourier image inspection [4] of the emission of the device to analyse the divergence angles of the OAM beams, as shown in Fig.1.

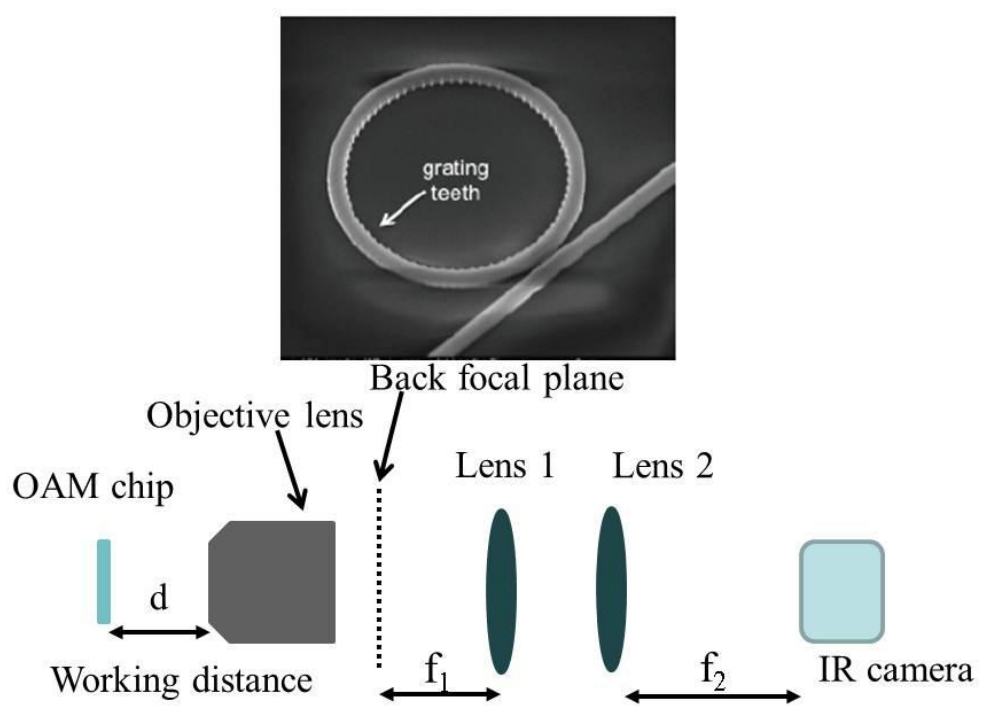

Fig. 1: Optical setup for Fourier image inspection of OAM beams.

The device is fabricated on silicon-on-insulator (SOI) substrate, with 220-nm thick silicon as the waveguide layer, and the SEM of one device is dipicted in the inset of Fig.1. According to the phase matching condition, the OAM vector mode order, $l$, is defined by $l=p-q$, where $p$ is the number of optical periods of the WGM inside the ring and $q$ is the number of grating elements[3]. The microring device ( $\mathrm{R}=19 \mu \mathrm{m}, \mathrm{q}=184$ ) has been characterized by launching continuous-wave light from a tunable laser into the access waveguide to excite the quasi-TE mode. The emission spectrum of the device is shown in Fig. 2. Each resonance corresponds to a distinctive WGM ( $p$ value). For detection of the angular emission, we recorded the images of the intensity distribution on the back focal plane of an objective (Olympus) on an infrared camera. These Fourier-plane images (momentum space) contain the angular information of the OAM emission. As dipicted in Fig.1, the experimental setup is a modified 4-f system. The OAM chip is placed at the working distance of the objective. A lens (lens 1 ) is placed at a distance $f_{1}$, the same as its focal length, from the back focal plane of the objective. Another lens (lens 2) is placed in front of the IR camera, and the distance between the lens 2 
and the camera is equal to the focal length of lens 2 . The system was calibrated using a commercial reflection grating with a known lattice parameter.

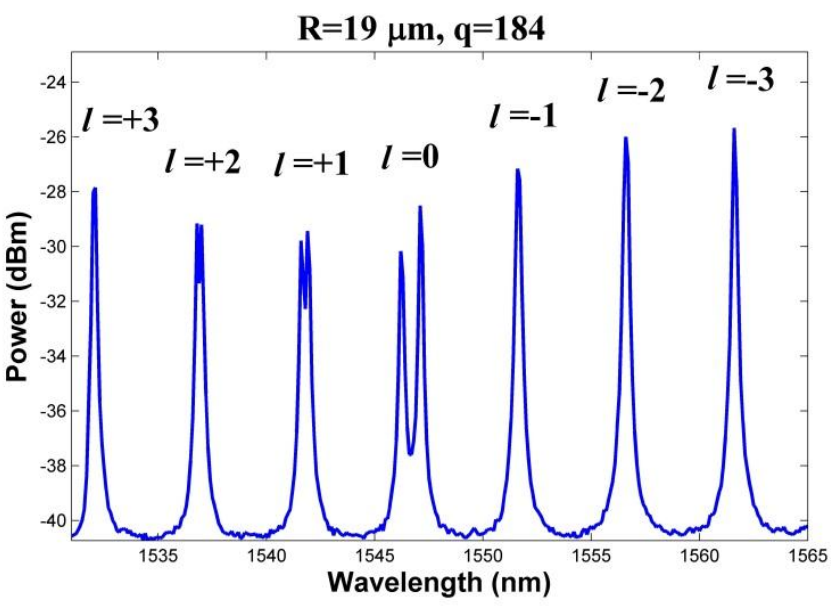

Fig. 2: Measured radiation spectrum for a device with $R=19 \mu \mathrm{m}$ measured by scanning input laser wavelength.

Fig.3(a) shows measured and calculated Fourier images of the OAM beam with the OAM order of $l=0$ and $l=1$. It should be noted that the emitted beams are vector beams with spatial-variant-polarization, which is the reason of the bright spot in the middle of the Fourier image of the beam with $l=1$ [5]. The measured results, show good agreement with the theoretical prediction calculated from a dipole-emission-based semianalytical model [5]. Fig.3(b) shows the calculated angluar distribution of the Fourier image. Fig. 3 (c) depicts the angles where the highest intensities are measured, which shows a good agreement with the theory.

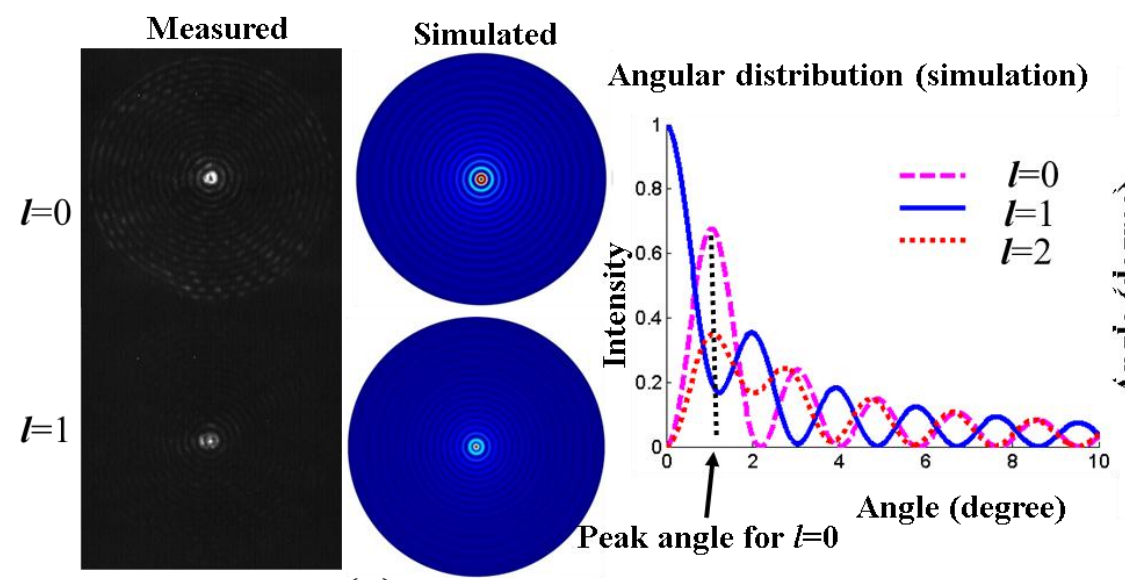

(a) (b)

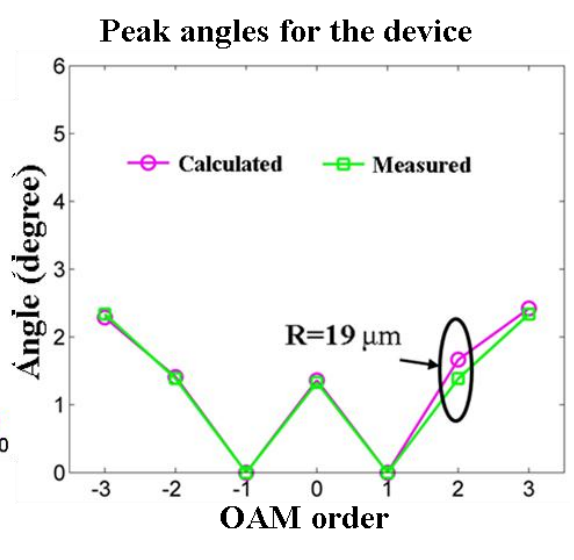

(c)

Fig. 3: (a) Measured and simulated fourier image (b)Simulated angular distribution (c) Peak angles for various OAM orders

In conclusion, we reported a method to measure the divergence angle of the OAM beams emited from integred OAM devices. The measured angular emission of the optical vortex beams are in excellent agreement with results obtained with a dipole-emission-based semianalytical model.

\section{References:}

[1] L. Allen, M. W. Beijersbergen, R. J. C. Spreeuw, and J. P. Woerdman, "Orbital angular momentum of light and the transformation of LaguerreGaussian laser modes," Phys. Rev. A vol. 45, no. 11, pp. 8185-8190 Jan. 1992.

[2] M. Padgett and R. Bowman, "Tweezers with a twist," Nat. Photonics, vol. 5, no. 6, pp. 343-348, Jun. 2011.

[3] X. Cai, J. Wang, M. J. Strain, B. Johnson-Morris, J. Zhu, M. Sorel, J. L. O’Brien, M. G. Thompson, and S. Yu, "Integrated compact optical vortex beam emitters.," Science, vol. 338, no. 6105, pp. 363-6, Oct. 2012.

[4] J. F. Galisteo-López, M. López-García, A. Blanco, C. López, "Studying Light Propagation in Self-Assembled Hybrid Photonic-Plasmonic Crystals by Fourier Microscopy," Langmuir vol. 28, no. 24, pp. 9174-9179, Apr. 2012.

[5] J. Zhu, X. Cai, Y. Chen, and S. Yu, "Theoretical model for angular grating-based integrated optical vortex beam emitters," Opt. Lett., vol. 38, no. 8, pp. 1343-1345, Mar. 2003. 